\title{
Generalized formulation of an encryption system based on a joint transform correlator and fractional Fourier transform
}

\author{
Juan M. Vilardy ${ }^{1}$, Yezid Torres ${ }^{2}$, María S. Millán ${ }^{1}$, and \\ Elisabet Pérez-Cabré ${ }^{1}$ \\ ${ }^{1}$ Applied Optics and Image Processing Group, Department of Optics and \\ Optometry, Universitat Politècnica de Catalunya, 08222 Terrassa (Barcelona), Spain \\ ${ }^{2}$ GOTS - Grupo de Óptica y Tratamiento de Señales, Physics School, Science \\ Faculty, Universidad Industrial de Santander, 678 Bucaramanga, Colombia \\ E-mail: juan.manuel.vilardy@estudiant.upc.edu
}

\begin{abstract}
We propose a generalization of the encryption system based on double random phase encoding (DRPE) and a joint transform correlator (JTC), from the Fourier domain to the fractional Fourier domain (FrFD) by using the fractional Fourier operators, such as the fractional Fourier transform (FrFT), fractional traslation, fractional convolution and fractional correlation. Image encryption systems based on a JTC architecture in the FrFD usually produce low quality decrypted images. In this work, we present two approaches to improve the quality of the decrypted images, which are based on nonlinear processing applied to the encrypted function (that contains the joint fractional power spectrum, JFPS) and the nonzero-order JTC in the FrFD. When the two approaches are combined, the quality of the decrypted image is higher. In addition to the advantages introduced by the implementation of the DRPE using a JTC, we demonstrate that the proposed encryption system in the FrFD preserves the shift-invariance property of the JTC-based encryption system in the Fourier domain, with respect to the lateral displacement of both the key random mask in the decryption process and the retrieval of the primary image. The feasibility of this encryption system is verified and analyzed by computer simulations.
\end{abstract}

Keywords: Encryption and decryption systems, joint transform correlator, double random phase encoding, fractional Fourier transform, fractional traslation, fractional convolution, fractional correlation, and nonlinear image processing. 


\section{Introduction}

Optical techniques are well-known to be suited for image encryption [1], since Réfrégier and Javidi proposed the method of double-random phase encoding (DRPE) [2], which has been further extended from the Fourier domain to the Fresnel domain $[3,4]$ and the fractional Fourier domain (FrFD) $[5,6,7,8,9]$, in order to increase the security of the DRPE technique. The DRPE generates the encrypted image, consisting of a stationary white noise image, for which two random phase masks (RPMs) in both the input plane and the Fourier plane are used [2]. The first optical setup of the DRPE technique was implemented using a classical $4 f$-processor [10]. Since this optical processor is a holographic system, it requires a strict optical alignment and, in addition to this, the decryption process needs the exact complex conjugate of one of the RPMs used as key. In order to mitigate these constraints, the joint transform correlator (JTC) architecture has been used to implement the DRPE technique in the Fourier domain [11, 12, 13, 14, 15]. The encrypted image for the JTC architecture is a real-valued distribution that is captured by a CCD camera in the Fourier plane while the DRPE implemented with a $4 f$-processor requires the recording of complex-valued information. The key mask used in the JTC-based encryption system is the same as for the decryption process [11].

Initially, the JTC-based encryption system has two choices for the security key: the first choice, the security key is designed to be the inverse Fourier transform of a RPM, just as it was proposed in Ref. [11], and the second choice, the security key is the RPM itself, just as it was proposed in $[12,13,14]$. For the first choice, the security key is a fully complex-valued distribution at the input plane of the JTC and, in order to optically reproduce this security key, the optical entrance of the setup proposed in [11] was split into two beams. This solution became more complex and required finer alignment than a conventional JTC. In Ref. [15], the authors proposed a different solution for this first choice, they represent the security key as a real-valued distribution whose Fourier transform had a uniform amplitude distribution and a uniformly random phase distribution. In the second choice, the security key is a random phase-only distribution at the input plane of the JTC. For this case, the security key can be easily implemented using a simple diffuser glass (random phase element) [12, 14].

The DRPE implemented with a JTC architecture has also been extended from the Fourier domain to the Fresnel domain [16, 17] and the FrFD [18, 19, 20, 21, 22]. The JTC-based encryption systems in the FrFD presented in $[18,19,20]$ are generalizations of the encryption system proposed in $[12,13]$. These encryption systems in the FrFD produce low quality decrypted images. The other optical security systems introduced in $[21,22]$ are based on the phase-shifting method, iterative processes and phase retrieval algorithms, and therefore, the image encryption and the decryption system differ from the DRPE proposed in $[2,5,11]$.

The cryptanalysis of the DRPE has proved that this security system is vulnerable to chosen-plaintext attacks (CPA) [23, 24], and known-plaintext attacks (KPA) [24, 25]. This weakness is due to the linear property of the DRPE system [24]. The DRPE 
implemented with a JTC is also vulnerable to CPA [26], and KPA [27]. These plaintext attacks can be extended to the DRPE systems in the FrFD, provided the fractional order of the fractional Fourier transform (FrFT) [28] is known.

Recently, the sparse representation $[29,30]$ and the photon-counting technique [31, 32, 33] have been integrated to the DRPE for information encoding and authentication. These integrations introduce a new level of information protection that increases the security of the DRPE and makes the authentication system more robust against unauthorized attacks [31, 32]. The sparse optical security system presented in [30] was described in the FrFD and it can be implemented using a JTC architecture [29].

In this paper, we propose a generalization of the JTC-based encryption systems described in [14] using the fractional Fourier operators, such as the FrFT, fractional traslation, and the new definitions for: fractional convolution and fractional correlation [34], with the purpose of improving the quality of the decrypted images and increasing the security of the encryption system in comparison with the previous encryption systems based on a JTC architecture $[11,12,13,14,15,18,19,20]$. We explain the main causes of the low quality of the decrypted images obtained in $[18,19,20]$ and propose two approaches to improve the quality of the decrypted images. The first approach introduces a simple nonlinear operation in the encrypted function that contains the joint fractional power spectrum (JFPS). The second approach combines the nonzero-order JTC [35] in the FrFD and the nonlinear operation presented in the first approach. The proposed encryption system keeps the properties of the JTC-based encryption systems that operate in the FrFD, such as new degrees of freedom for the optical setup, because the position of the lens in the proposed optical encryption setup can be chosen, so that an additional key given by the fractional order of the FrFT is introduced in the security system. This additional key improves security.

The encryption system introduced here, can be implemented using a simplified JTC in the FrFD that avoids the beam splitting required by other optical JTC implementations $[11,18,19,20]$. In addition, the two approaches used to improve the quality of the decrypted image do not increase the amount of information to be transmitted because the resulting encrypted function has the same size as the original version. The proposed JTC-based encryption-decryption system in the FrFD preserves the shift-invariance property with respect to lateral displacements of both the key random mask in the decryption process and the retrieval of the primary image $[1,34]$.

The remainder of this paper is organized as follows: In section 2, a JTC-based encryption system using fractional Fourier operators is introduced and the reasons of the low quality of the decrypted image are analyzed. In section 3, two approaches to improve the quality of the decrypted image are presented and also, the simulation results to demonstrate the feasibility of the modified encryption and decryption system are given. Conclusions are outlined in section 4 . 


\section{Image encryption system based on the JTC architecture and fractional Fourier transform}

In this section, we generalize the encryption system presented in section 2 of Ref. [14] using fractional Fourier operators, such as the FrFT (Appendix A), fractional traslation (Appendix B), fractional convolution (Appendix C) and fractional correlation (Appendix $\mathrm{C}$ ). Let $f(x)$ be the original image to be encrypted with real values in the interval $[0,1]$, written in one-dimensional notation for the sake of simplicity, and $r(x)$ and $h(x)$ be two RPMs given by

$$
r(x)=\exp \{i 2 \pi s(x)\}, \quad h(x)=\exp \{i 2 \pi n(x)\},
$$

where $s(x)$ and $n(x)$ are normalized positive functions randomly generated, statistically independent and uniformly distributed in the interval $[0,1]$. In order to simplify the following equations, we define a new function $g(x)=f(x) r(x)$, which is the original image to be encrypted bonded to the RPM $r(x)$.

For the encryption system shown in Fig. 1 (Part I), the new function $g(x)$ and the RPM $h(x)$ are placed side by side at the input plane of the JTC by means of the fractional traslation operators $\mathscr{T}_{a ; \alpha}$ and $\mathscr{T}_{-a ; \alpha}$, respectively, where $a$ is a real value and $\alpha$ represents the fractional order of the FrFT operator to be used. Therefore, the input plane of the JTC-based encryption system is

$$
\begin{aligned}
t(x)= & \mathscr{T}_{a ; \alpha}[g(x)]+\mathscr{T}_{-a ; \alpha}[h(x)] \\
= & g(x-a) \exp \left\{i 2 \pi a\left(x-\frac{a}{2}\right) \cot \alpha\right\} \\
& +h(x+a) \exp \left\{-i 2 \pi a\left(x+\frac{a}{2}\right) \cot \alpha\right\} .
\end{aligned}
$$

The JFPS, also named the encrypted fractional power spectrum $e_{\alpha}(u)$, is given by:

$$
\begin{aligned}
e_{\alpha}(u)= & \operatorname{JFPS}_{\alpha}(u)=\left|\mathscr{F}^{\alpha}\{t(x)\}\right|^{2} \\
= & \left|\mathscr{F}^{\alpha}\left\{\mathscr{T}_{a ; \alpha}[g(x)]+\mathscr{T}_{-a ; \alpha}[h(x)]\right\}\right|^{2} \\
= & \left|g_{\alpha}(u) \exp \{i 2 \pi a u \csc \alpha\}+h_{\alpha}(u) \exp \{-i 2 \pi a u \csc \alpha\}\right|^{2} \\
= & \left|g_{\alpha}(u)\right|^{2}+\left|h_{\alpha}(u)\right|^{2}+g_{\alpha}^{*}(u) h_{\alpha}(u) \exp \{-i 2 \pi(2 a) u \csc \alpha\} \\
& +g_{\alpha}(u) h_{\alpha}^{*}(u) \exp \{i 2 \pi(2 a) u \csc \alpha\},
\end{aligned}
$$

where the superscript ${ }^{*}$ denotes the complex conjugation operation. The pure linear phase terms symmetrically introduced in Eq. (2) are used to ensure the complete overlapping of the fractional spectra corresponding to $g_{\alpha}(u)=\mathscr{F}^{\alpha}\{g(x)\}$ and $h_{\alpha}(u)=$ $\mathscr{F}^{\alpha}\{h(x)\}$ in Eq. (3). The encrypted image $e_{\alpha}(u)$ is a real-valued distribution that is acquired by a CCD camera. The security keys of the encryption system are the RPM $h(x)$ and the fractional order $\alpha$ (the distances $d_{1}, d_{2}$ and the focal length of the lens, control the value of the fractional order $\alpha[28,36])$. The RPM $r(x)$ is used to spread the information content of the original image $f(x)$ onto the encrypted image $e_{\alpha}(u)$. When the fractional order is equal to $\pi / 2$, the Eq. (3) is reduced to the Eq. (2) of Ref. [14].

In the decryption system (Fig. 1, part II), the RPM $h(x)$ is shifted to $x=-a$ with

fractional order $\alpha$ and, consequently, the encrypted image $e_{\alpha}(u)$ located in the FrFD is 


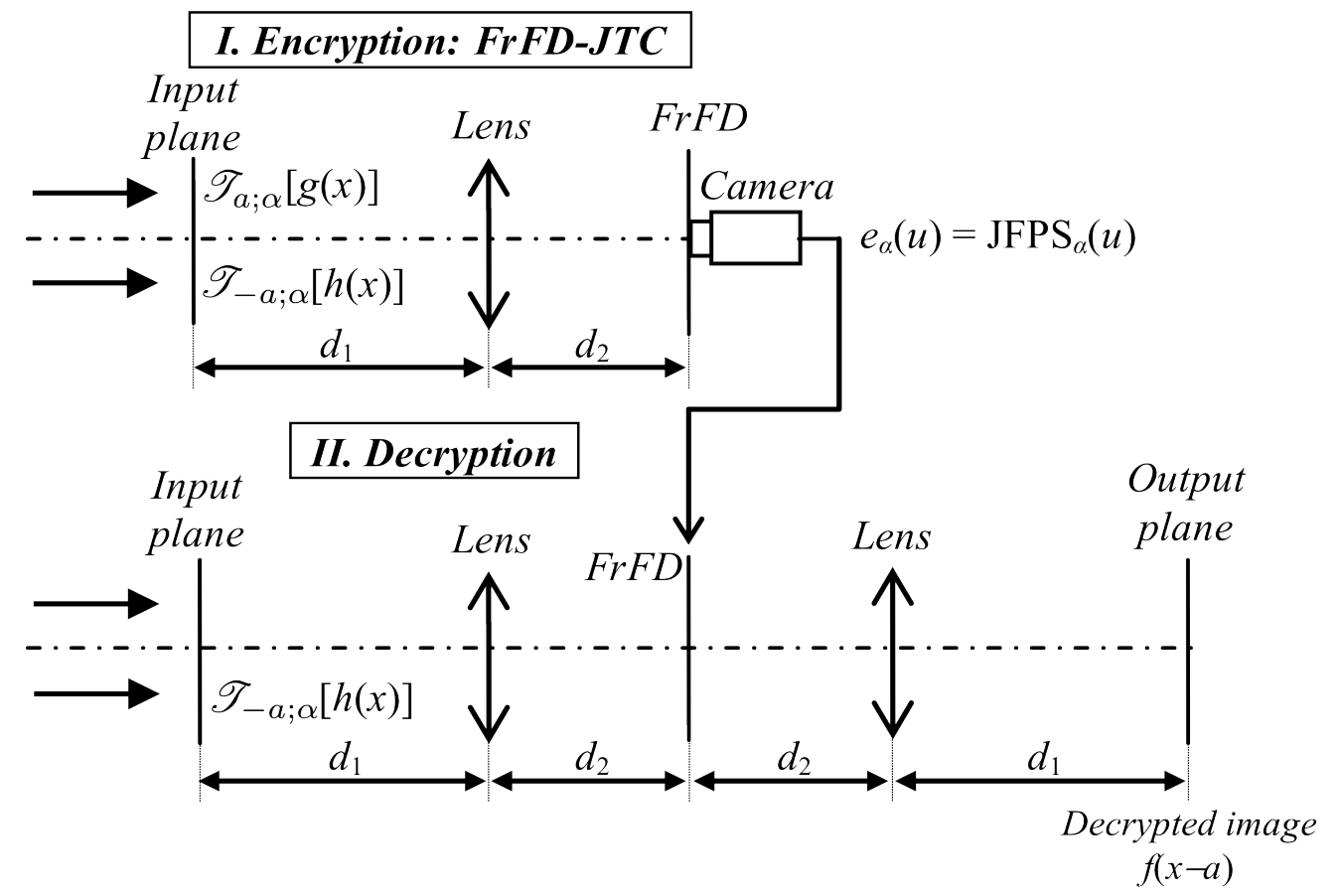

Figure 1. Schematic representation of the optical setup. The encryption system (Part I) is based on a JTC in the FrFD and the decryption system (Part II) is composed by two successive FrFTs.

illuminated by $\mathscr{F}^{\alpha}\left\{\mathscr{T}_{-a ; \alpha}[h(x)]\right\}$. Using the results of Appendix B and Eq. (3), this initial step of the decryption process can be expressed by

$$
\begin{aligned}
d_{\alpha}(u)= & e_{\alpha}(u) \mathscr{F}^{\alpha}\left\{\mathscr{T}_{-a ; \alpha}[h(x)]\right\}=e_{\alpha}(u) h_{\alpha}(u) \exp \{-i 2 \pi a u \csc \alpha\} \\
= & g_{\alpha}(u) g_{\alpha}^{*}(u) \exp \left\{-i \pi u^{2} \cot \alpha\right\} \\
& \times h_{\alpha}(u) \exp \left\{i \pi u^{2} \cot \alpha\right\} \exp \{-i 2 \pi a u \csc \alpha\} \\
& +h_{\alpha}(u) h_{\alpha}^{*}(u) \exp \left\{-i \pi u^{2} \cot \alpha\right\} \\
& \times h_{\alpha}(u) \exp \left\{i \pi u^{2} \cot \alpha\right\} \exp \{-i 2 \pi a u \csc \alpha\} \\
& +h_{\alpha}(u) h_{\alpha}(u) \exp \left\{i \pi u^{2} \cot \alpha\right\} \\
& \times g_{\alpha}^{*}(u) \exp \left\{-i \pi u^{2} \cot \alpha\right\} \exp \{-i 2 \pi(3 a) u \csc \alpha\} \\
& +h_{\alpha}(u) h_{\alpha}^{*}(u) \exp \left\{-i \pi u^{2} \cot \alpha\right\} \\
& \times g_{\alpha}(u) \exp \left\{i \pi u^{2} \cot \alpha\right\} \exp \{i 2 \pi a u \csc \alpha\} .
\end{aligned}
$$

The FrFT at fractional order $-\alpha$ of Eq. (4) is

$$
\begin{aligned}
d(x)= & \mathscr{F}^{-\alpha}\left\{d_{\alpha}(u)\right\}=\mathscr{T}_{-a ; \alpha}\left[\left\{g(x) \circledast_{\alpha} g(x)\right\} *_{\alpha} h(x)\right] \\
& +\mathscr{T}_{-a ; \alpha}\left[\left\{h(x) \circledast_{\alpha} h(x)\right\} *_{\alpha} h(x)\right]+\mathscr{T}_{-3 a ; \alpha}\left[\left\{h(x) *_{\alpha} h(x)\right\} \circledast_{\alpha} g(x)\right] \\
& +\mathscr{T}_{a ; \alpha}\left[\left\{h(x) \circledast_{\alpha} h(x)\right\} *_{\alpha} g(x)\right],
\end{aligned}
$$

where $*_{\alpha}$ indicates the fractional convolution operator and $\circledast_{\alpha}$ denotes the fractional correlation operator. The first, second, and third terms of Eq. (5) are spatially separated noisy images at coordinates $x=-a$ and $x=-3 a$. The fourth term on the right side of 
Eq. (5) retains the information to be decrypted [14]. Therefore, if we take the absolute value of this term, the decrypted image $\hat{f}(x)$ at coordinate $x=a$ is

$$
\hat{f}(x-a)=\left|\mathscr{T}_{a ; \alpha}\left[\left\{h(x) \circledast_{\alpha} h(x)\right\} *_{\alpha}\{f(x) r(x)\}\right]\right| .
$$

The decrypted image $\hat{f}(x)$ would no longer be the original image $f(x)$, because the fractional autocorrelation of the RPM $h(x)$ in general is not equal to a Dirac delta function $\delta(x)$. This fact is the principal cause of the low quality of the obtained decrypted images in the encryption-decryption systems proposed in Refs. [18, 19]. For the decryption system presented in Ref. [20], the cause of the low quality of the decrypted images is the consideration that the autocorrelation of a RPM can be approximated by a Dirac delta distribution $\delta(x)$, this consideration is not longer true for the DRPE technique just as it was demonstrated in Ref. [14]. The Eq. (6) is a fractional Fourier generalization of the Eq. (4) of Ref. [14].

The simulation results for the encryption-decryption system presented in this section are shown in Fig. 2. The original image to be encrypted $f(x)$ and the random distribution code $n(x)$ of the RPM $h(x)$ are depicted in Figs. 2(a) and 2(b), respectively. The encrypted image $e_{\alpha}(u)$ for the fractional order $p=1.5(\alpha=p \pi / 2=3 \pi / 4)$ is displayed in Fig. 2(c). The absolute value of the output plane for the decryption procedure $|d(x)|$ with the correct keys, the fractional order $p$ and the RPM $h(x)$, is shown in Fig. 2(d). The decrypted image $\tilde{f}(x)$ presented in Fig. 2(e) is the magnified region of interest, centered at position $x=a$, of the output plane $|d(x)|$, this image $\tilde{f}(x)$ has been obtained through the whole process represented by Eqs. (2)-(5). The decrypted image $\hat{f}(x)$ shown in Fig. $2(\mathrm{f})$ has been obtained by calculating just the right term of Eq. (6). The fractional autocorrelation of the RPM $h(x)$ with $\alpha=3 \pi / 4$ is shown in Figs. 2(g)-2(i): Figure 2(g) represents the modulus $\left|h(x) \circledast_{\alpha} h(x)\right|$ in a linear scale, Fig. 2(h) is the phase $h(x) \circledast_{\alpha} h(x) /\left|h(x) \circledast_{\alpha} h(x)\right|$ coded in grey levels, and Fig. 2(i) shows a pseudocolor three-dimensional representation of the modulus $\left|h(x) \circledast_{\alpha} h(x)\right|$.

The decrypted images shown in Figs. 2(e) and 2(f) are poor quality because the fractional autocorrelation of the RPM $h(x)$ is a noisy image (see Figs. 2(g)-2(i)), this fact was determined by the result of Eq. (6). To quantitatively evaluate the quality of the decrypted images, we use the root mean square error (RMSE) [37]. The RMSE for the decrypted images $\tilde{f}(x)$ and $\hat{f}(x)$, with respect to the original image $f(x)$ is defined using the following expression

$$
\operatorname{RMSE}=\left(\frac{\sum_{x=1}^{M}[f(x)-\breve{f}(x)]^{2}}{\sum_{x=1}^{M}[f(x)]^{2}}\right)^{\frac{1}{2}},
$$

where $\mathrm{RMSE}_{1}$ is defined for $\breve{f}(x)=\tilde{f}(x)$ and $\mathrm{RMSE}_{2}$ for $\breve{f}(x)=\hat{f}(x)$. It is worth remarking that the decrypted images $\tilde{f}(x)$ and $\hat{f}(x)$ were obtained in two different ways. In Fig. 3, we present the results for the $\mathrm{RMSE}_{1}$ and $\mathrm{RMSE}_{2}$ versus the fractional order $p$. When $p=0$, the FrFT operator corresponds to the identity transform and the RMSE is zero in Fig. 3, this particular fractional order $p=0$ is trivial and makes no sense, so we skip it for the encryption system. The minimum value different from 
zero for the RMSE curves in Fig. 3, is 0.509 that corresponds to the fractional orders $p= \pm 1$ (direct and inverse Fourier transform, respectively), this case was analyzed and reported in Ref. [14]. When the fractional order is different from $p= \pm 1$ or $p=0$ in Fig. 3, the range of values for the RMSE curves are between 0.6 and 0.8 . These high values of RMSE confirm the very low quality of the decrypted images for different fractional orders.

\section{Approaches to improve the quality of the decrypted image}

We propose two approaches in order to improve the quality of the decrypted image in the encryption-decryption system presented in section 2. The first approach introduces a simple nonlinear operation on the JFPS. The second approach combines the nonzeroorder JTC $[35,38]$ in the FrFD and the nonlinear operation of the first approach.

\subsection{Approach I: Nonlinear modification of the JTC architecture}

In section 2, we have demonstrated that the fractional autocorrelation of the RPM $h(x)$ presented in Eq. (6) significantly degrades the quality of the decrypted image. Therefore, to eliminate this fractional autocorrelation from Eq. (6), we propose to modify the encrypted function (the JFPS given by Eq. (3)) by extending the nonlinear method presented in Ref. [14] to the FrFD. Thus, the new encrypted function $e_{\alpha}^{N_{1}}(u)$ is defined as the JFPS divided by the nonlinear term $\left|h_{\alpha}(u)\right|^{2}$, and it is represented by the following equation

$$
\begin{aligned}
e_{\alpha}^{N_{1}}(u)= & \frac{\operatorname{JFPS}_{\alpha}(u)}{\left|h_{\alpha}(u)\right|^{2}}=\frac{\left|g_{\alpha}(u)\right|^{2}}{\left|h_{\alpha}(u)\right|^{2}}+1+g_{\alpha}^{*}(u) \frac{h_{\alpha}(u)}{\left|h_{\alpha}(u)\right|^{2}} \exp \{-i 2 \pi(2 a) u \csc \alpha\} \\
& +g_{\alpha}(u) \frac{h_{\alpha}^{*}(u)}{\left|h_{\alpha}(u)\right|^{2}} \exp \{i 2 \pi(2 a) u \csc \alpha\}
\end{aligned}
$$

If $\left|h_{\alpha}(u)\right|^{2}$ is equal to zero for a particular value of $u$, this intensity value is substituted by a very small constant to avoid singularities when computing $e_{\alpha}^{N_{1}}(u)$. The new encrypted function remains as a real-valued function that can be computed from the intensity distributions of the $\operatorname{JFPS}_{\alpha}(u)$ and $\left|h_{\alpha}(u)\right|^{2}$, previously acquired by the CCD camera. The Eq. (8) is also a fractional Fourier generalization of the Eq. (8) of Ref. [14].

For the decryption system, we have the product between the new encrypted image $e_{\alpha}^{N_{1}}(u)$ and the FrFT at fractional order $\alpha$ of $\mathscr{T}_{-a ; \alpha}[h(x)]$ as

$$
\begin{aligned}
d_{\alpha}^{N_{1}}(u)= & e_{\alpha}^{N_{1}}(u) \mathscr{F}^{\alpha}\left\{\mathscr{T}_{-a ; \alpha}[h(x)]\right\}=e_{\alpha}^{N_{1}}(u) h_{\alpha}(u) \exp \{-i 2 \pi a u \csc \alpha\} \\
= & \left|g_{\alpha}(u)\right|^{2} \frac{h_{\alpha}(u)}{\left|h_{\alpha}(u)\right|^{2}} \exp \{-i 2 \pi a u \csc \alpha\}+h_{\alpha}(u) \exp \{-i 2 \pi a u \csc \alpha\} \\
& +g_{\alpha}^{*}(u) \frac{h_{\alpha}^{2}(u)}{\left|h_{\alpha}(u)\right|^{2}} \exp \{-i 2 \pi(3 a) u \csc \alpha\} \\
& +g_{\alpha}(u) \frac{h_{\alpha}(u) h_{\alpha}^{*}(u)}{\left|h_{\alpha}(u)\right|^{2}} \exp \{i 2 \pi a u \csc \alpha\}
\end{aligned}
$$




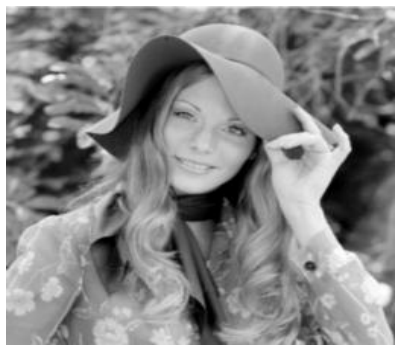

(a)

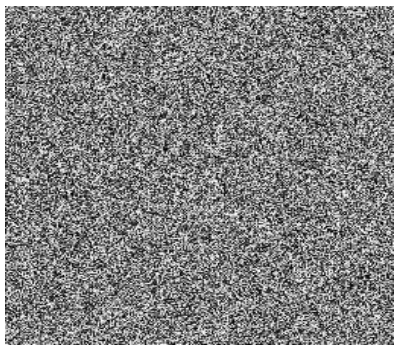

(b)

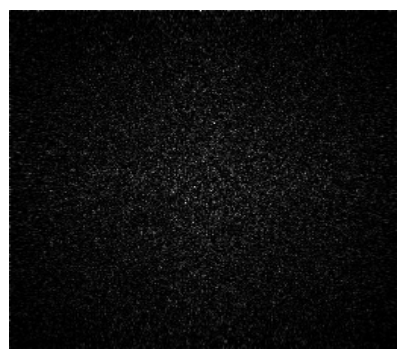

(c)

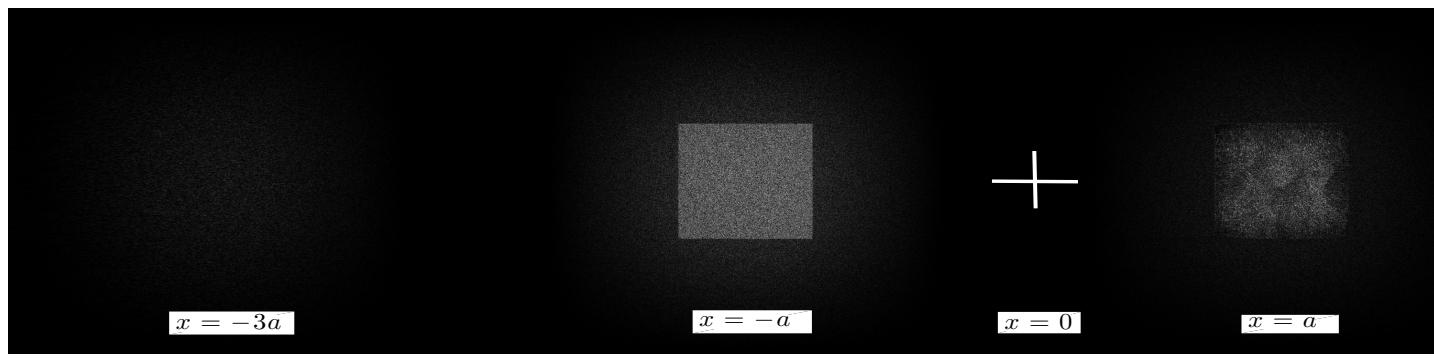

(d)

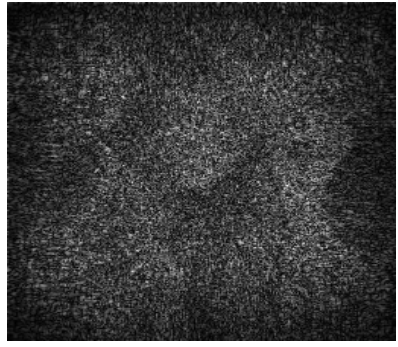

(e)

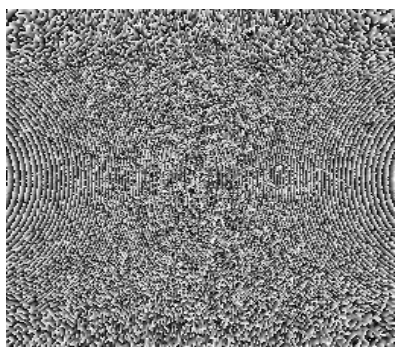

(h)

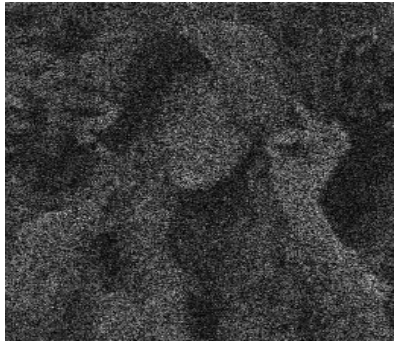

(f)

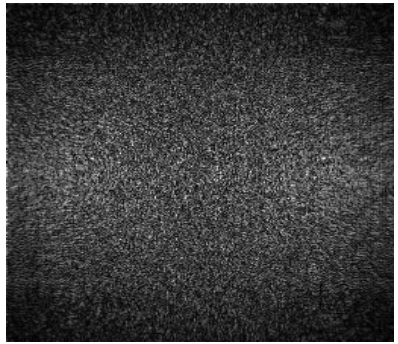

$(\mathrm{g})$

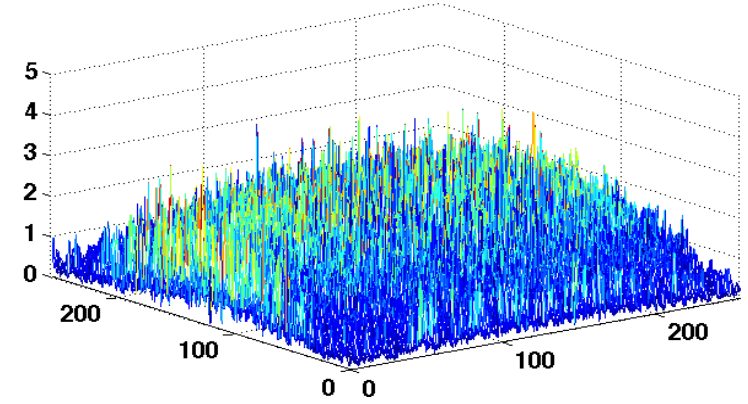

(i)

Figure 2. (a) Original image to be encrypted $f(x)$, (b) Random distribution code $n(x)$ of the RPM $h(x)$, (c) Encrypted image $e_{\alpha}(u)$ for the fractional order $p=1.5$ $(\alpha=p \pi / 2=3 \pi / 4)$, (d) Absolute value of the output plane $|d(x)|$ for the decryption system with the correct keys, the fractional order $p$ and the RPM $h(x)$. (e) Magnified region of interest of $|d(x)|$ corresponding to the decrypted image $\tilde{f}(x)$ at coordinate $x=a$ and, (f) Decrypted image $\hat{f}(x)$ using just the right term of Eq. (6). Fractional autocorrelation of $h(x)$ with $\alpha=3 \pi / 4$ : (g) modulus $\left|h(x) \circledast_{\alpha} h(x)\right|$ in a linear scale, (h) phase $h(x) \circledast_{\alpha} h(x) /\left|h(x) \circledast_{\alpha} h(x)\right|$ coded in grey levels, and (i) pseudocolor threedimensional representation of the modulus $\left|h(x) \circledast_{\alpha} h(x)\right|$. 


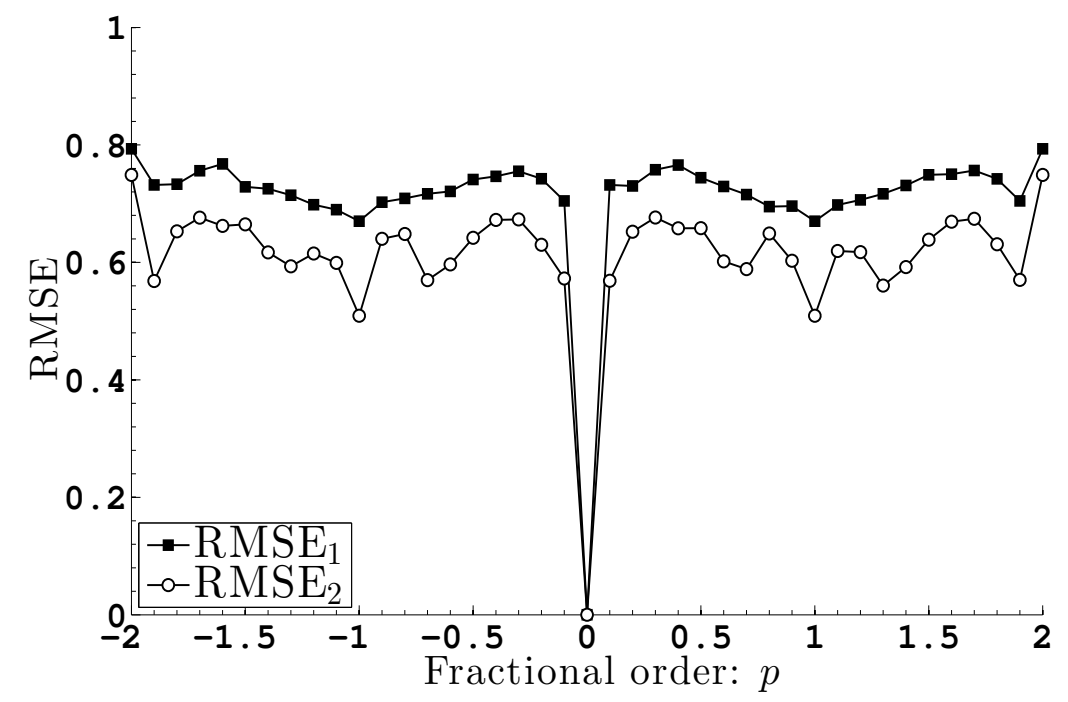

Figure 3. RMSE $E_{1}$ and $\mathrm{RMSE}_{2}$ versus the fractional order $p$ for the case presented in Fig. 2.

To retrieve the original image, we apply the FrFT operator at fractional order $-\alpha$ to the simplified fourth term of Eq. (9) and then, an absolute value function. Therefore, the decrypted image obtained at coordinate $x=a$ is given by

$$
\begin{aligned}
\hat{f}(x-a) & =\left|\mathscr{F}^{-\alpha}\left[g_{\alpha}(u) \exp \{i 2 \pi a u \csc \alpha\}\right]\right| \\
& =\left|\mathscr{T}_{a ; \alpha}[f(x) r(x)]\right|=f(x-a) .
\end{aligned}
$$

The nonlinear operation introduced in the Eq. (8) allows the retrieval of the original image in the decryption system. Unlike Eq. (6), the result of Eq. (10) does not have the fractional autocorrelation of the RPM $h(x)$, and thus, the quality of the decrypted image would significantly increase.

In Fig. 4, we present the results of the numerical simulations for the nonlinear JTC-encryption system in the FrFD proposed in this subsection. The original image $f(x)$ to be encrypted is shown in Fig. 4(a). The new encrypted image $e_{\alpha}^{N_{1}}(u)$ for the fractional order $p=1.5$ is presented in Fig. 4(b). The absolute value of the output plane for the decryption procedure $\left|d^{N_{1}}(x)\right|=\left|\mathscr{F}^{-\alpha}\left\{d_{\alpha}^{N_{1}}(u)\right\}\right|$ with the true keys, the fractional order $p$ and the RPM $h(x)$, is displayed in Fig. 4(c). We observe in Fig. 4(c) that the component at coordinate $x=-a$ is more intense than the components at coordinates $x=-3 a$ and $x=a$ (decrypted image). The decrypted image $\tilde{f}(x)$ presented in Fig. $4(\mathrm{~d})$ is the magnified region of interest, centered at position $x=a$, of the output plane $\left|d^{N_{1}}(x)\right|$. The RMSE between the original image from Fig. 4(a) and the decrypted image from Fig. 4(d) is 0.187 . Due to the removal of the fractional autocorrelation term from the decrypted signal (compare Eq. (6) and Eq. (10)), the quality of the retrieved image in Fig. 4(d) is remarkably improved in comparison to the decrypted images shown in Figs. 2(e) and 2(f). If we visually compare the decrypted image obtained in Fig. 4(d) with respect to the original image to be encrypted and shown in Fig. 4(a), we can see some noise presented in the decrypted image of Fig. 4(d). This noise will be removed 
from the decrypted image in the next subsection. The noisy decrypted image displayed in Fig. 4(e), corresponds to the retrieved image in the decryption system when the key of the RPM $h(x)$ is wrong and the value of the fractional order is correct.

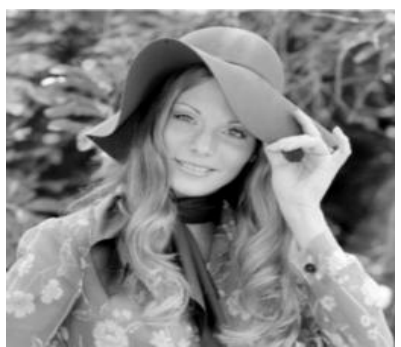

(a)

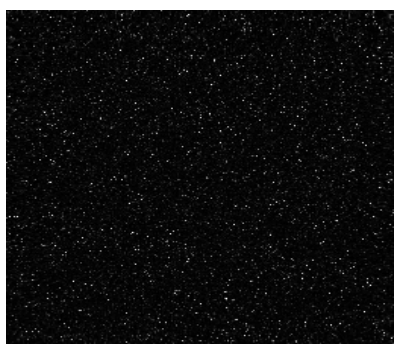

(b)

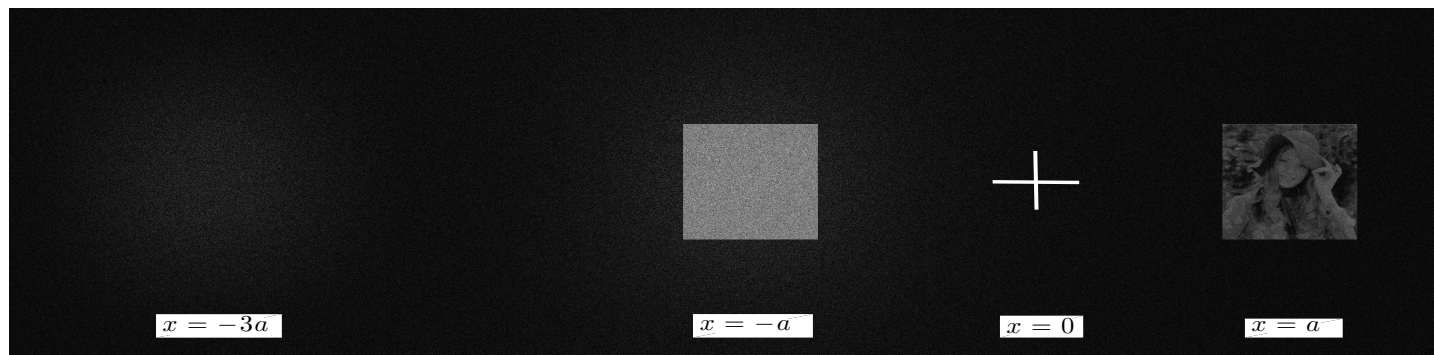

(c)

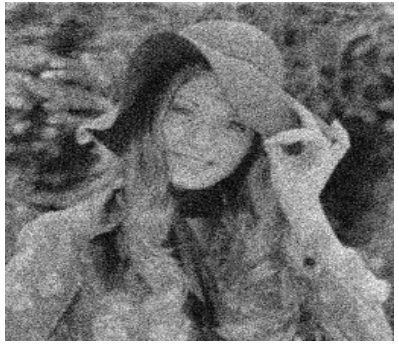

(d)

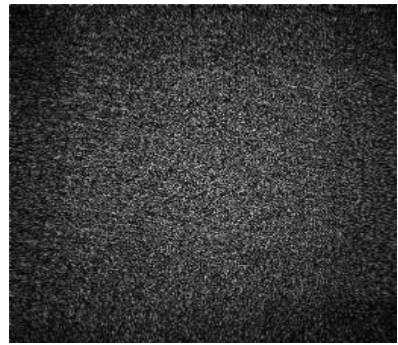

(e)

Figure 4. (a) Original image to be encrypted $f(x)$, (b) Encrypted image $e_{\alpha}^{N_{1}}(u)$ for the fractional order $p=1.5$, (c) Absolute value of the output plane $\left|d^{N_{1}}(x)\right|$ for the decryption system with the correct keys, the fractional order $p$ and the RPM $h(x)$. (d) Magnified region of interest of $\left|d^{N_{1}}(x)\right|$ corresponding to the decrypted image $\tilde{f}(x)$ at coordinate $x=a$ and (e) Decrypted image using an incorrect RPM $h(x)$ and the correct fractional order.

\subsection{Approach II: Removing the zero-order fractional power spectra from the JFPS}

The nonzero-order JTC was used to improve the detection efficiency of the conventional JTC in the image pattern recognition $[35,38]$. In this subsection, we propose to use a nonzero-order JTC in the FrFD and also, to apply the nonlinear operation introduced in the subsection 3.1 to further improve the quality of the decrypted image obtained in Fig. 4(d). 
In order to define the new encrypted image $e_{\alpha}^{N_{2}}(u)$, we eliminate the zero-order fractional power spectra $\left(\left|g_{\alpha}(u)\right|^{2}\right.$ and $\left|h_{\alpha}(u)\right|^{2}$ terms) of the JFPS by extending the nonzero-order JTC architecture to the FrFD. Thus, we define the encrypted image $e_{\alpha}^{N_{2}}(u)$ as the modified JFPS divided by the nonlinear term $\left|h_{\alpha}(u)\right|^{2}$

$$
\begin{aligned}
e_{\alpha}^{N_{2}}(u)= & \frac{\operatorname{JFPS}_{\alpha}(u)-\left|g_{\alpha}(u)\right|^{2}-\left|h_{\alpha}(u)\right|^{2}}{\left|h_{\alpha}(u)\right|^{2}} \\
= & g_{\alpha}^{*}(u) \frac{h_{\alpha}(u)}{\left|h_{\alpha}(u)\right|^{2}} \exp \{-i 2 \pi(2 a) u \csc \alpha\} \\
& +g_{\alpha}(u) \frac{h_{\alpha}^{*}(u)}{\left|h_{\alpha}(u)\right|^{2}} \exp \{i 2 \pi(2 a) u \csc \alpha\} .
\end{aligned}
$$

The encrypted function $e_{\alpha}^{N_{2}}(u)$ is still a real-valued function. We need to acquire three intensity distributions, which are the $\operatorname{JFPS}_{\alpha}(u),\left|g_{\alpha}(u)\right|^{2}$ and $\left|h_{\alpha}(u)\right|^{2}$ to compute the encrypted image $e_{\alpha}^{N_{2}}(u)$.

In the decryption process, we perform the product between the encrypted function $e_{\alpha}^{N_{2}}(u)$ and the FrFT at fractional order $\alpha$ of $\mathscr{T}_{-a ; \alpha}[h(x)]$, this product is given by

$$
\begin{aligned}
d_{\alpha}^{N_{2}}(u)= & e_{\alpha}^{N_{2}}(u) \mathscr{F}^{\alpha}\left\{\mathscr{T}_{-a ; \alpha}[h(x)]\right\}=e_{\alpha}^{N_{2}}(u) h_{\alpha}(u) \exp \{-i 2 \pi a u \csc \alpha\} \\
= & \frac{h_{\alpha}(u)}{\left|h_{\alpha}(u)\right|} \frac{h_{\alpha}(u)}{\left|h_{\alpha}(u)\right|} \exp \left\{i \pi u^{2} \cot \alpha\right\} \\
& \times g_{\alpha}^{*}(u) \exp \left\{-i \pi u^{2} \cot \alpha\right\} \exp \{i 2 \pi(-3 a) u \csc \alpha\} \\
& +\frac{h_{\alpha}(u) h_{\alpha}^{*}(u)}{\left|h_{\alpha}(u)\right|^{2}} g_{\alpha}(u) \exp \{i 2 \pi a u \csc \alpha\} .
\end{aligned}
$$

The FrFT at fractional order $-\alpha$ of last equation is

$$
\begin{aligned}
d^{N_{2}}(x) & =\mathscr{F}^{-\alpha}\left\{d_{\alpha}^{N_{2}}(u)\right\} \\
& =\mathscr{T}_{-3 a ; \alpha}\left[\left\{h_{1}(x) *_{\alpha} h_{1}(x)\right\} \circledast_{\alpha} g(x)\right]+\mathscr{T}_{a ; \alpha}[g(x)],
\end{aligned}
$$

where $h_{1}(x)=\mathscr{F}^{-\alpha}\left\{h_{\alpha}(u) /\left|h_{\alpha}(u)\right|\right\}$. When the absolute value is applied to the second term of Eq. (13), we obtain the decrypted image at coordinate $x=a$

$$
\hat{f}(x-a)=\left|\mathscr{T}_{a ; \alpha}[g(x)]\right|=\left|\mathscr{T}_{a ; \alpha}[f(x) r(x)]\right|=f(x-a) .
$$

This equation is equal to Eq. (10), and therefore, for both equations we expect a higher quality for the decrypted image in comparison with the retrieved image from Eq. (6) because the fractional autocorrelation term of the RPM $h(x)$ was removed from the right side of Eqs. (10) and (14). We remark that the output planes for the decryption system in the approaches I and II, $d^{N_{1}}(x)$ (it has four terms) and $d^{N_{2}}(x)$ (it has two terms), respectively, are very different.

The simulation results for the encryption-decryption system presented in this subsection are shown in Fig. 5. The original image $f(x)$ to be encrypted is displayed in Fig. 5(a). The encrypted image $e_{\alpha}^{N_{2}}(u)$ with the fractional order $p=1.5$ is presented in Fig. 5(b). The absolute value of the output plane for the decryption procedure $\left|d^{N_{2}}(x)\right|$ with the true keys, the fractional order $p$ and the RPM $h(x)$, is shown in Fig. 5(c). The decrypted image $\tilde{f}(x)$ depicted in Fig. $5(\mathrm{~d})$ is the magnified region of interest, centered 
at position $x=a$, of the output plane $\left|d^{N_{2}}(x)\right|$. The RMSE between the original image from Fig. 5(a) and the decrypted image from Fig. 5(d) is 0.012. The image quality for the decrypted image of Fig. 5(d) is higher than the decrypted image of Fig. 4(d), because the zero-order fractional power spectra were removed from the JFPS. We note in Fig. 5(c) that the decrypted image at coordinate $x=a$ is more intense in comparison with the decrypted image from Fig. 4(c) at the same coordinate, this fact is due to the removal of the zero-order fractional power from the JFPS. Therefore, the approach II is more efficient than the approach I with respect to the recovered intensity for the decrypted image.

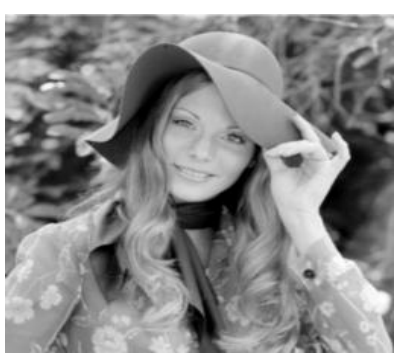

(a)

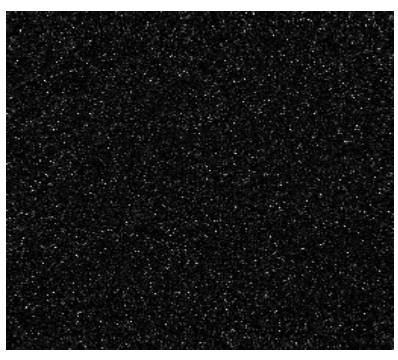

(b)

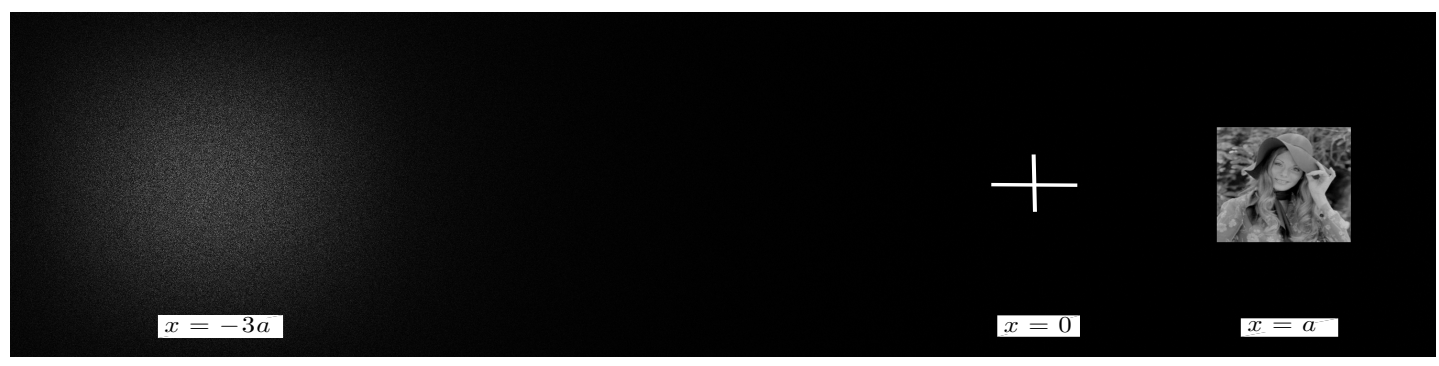

(c)

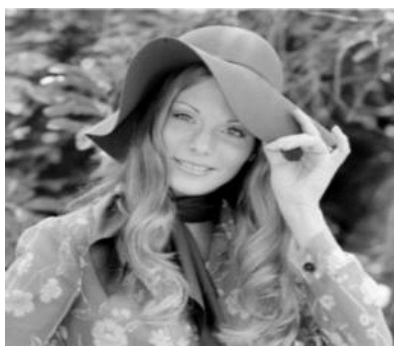

(d)

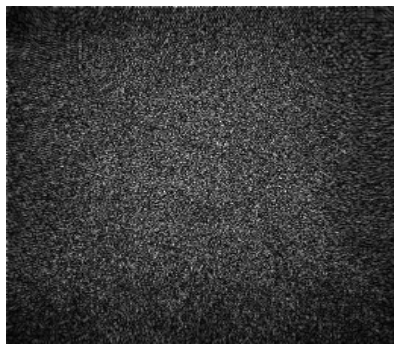

(e)

Figure 5. (a) Original image to be encrypted $f(x)$, (b) Encrypted image $e_{\alpha}^{N_{2}}(u)$ with the fractional order $p=1.5$, (c) Absolute value of the output plane $\left|d^{N_{2}}(x)\right|$ for the decryption system with the true keys, the fractional order $p$ and the RPM $h(x)$. (d) Magnified region of interest of $\left|d^{N_{2}}(x)\right|$ corresponding to the decrypted image $\tilde{f}(x)$ at coordinate $x=a$ and (e) Decrypted image using an incorrect fractional order $p=1.497$ and the correct RPM $h(x)$. 
The noisy decrypted image shown in Fig. 5(e) corresponds to the retrieved image in the decryption system when the key of the RPM $h(x)$ is correct and the value of the fractional order $p$ differs from the correct value in $0.2 \%$. When an incorrect RPM $h(x)$ or a wrong value of the fractional order $p$ are used in the decryption system, the decrypted images obtained are noisy patterns similar to Fig. 5(e). Therefore, the provided result demonstrate that the all keys (the RPM $h(x)$ and the fractional order $\alpha$ ) are required in the decryption system for the correct retrieval of the original image.

The sensitivity on the fractional order $p$ of the FrFT for the decrypted images is examined by introducing small error in this, and then we evaluate the RMSE ${ }_{1}$, which is defined in Eq. (7), between the original image $f(x)$ and the decrypted image $\tilde{f}(x)$ to measure the level of protection on the encrypted image $e_{\alpha}^{N_{2}}(u)$. Figure 6 presents the $\mathrm{RMSE}_{1}$ versus the relative error of $p$ for the image retrieval and it shows that $p$ is sensitive to a variation of $10^{-4}$. Therefore, the space key for the fractional order of the FrFT is $4 \times 10^{4}$.

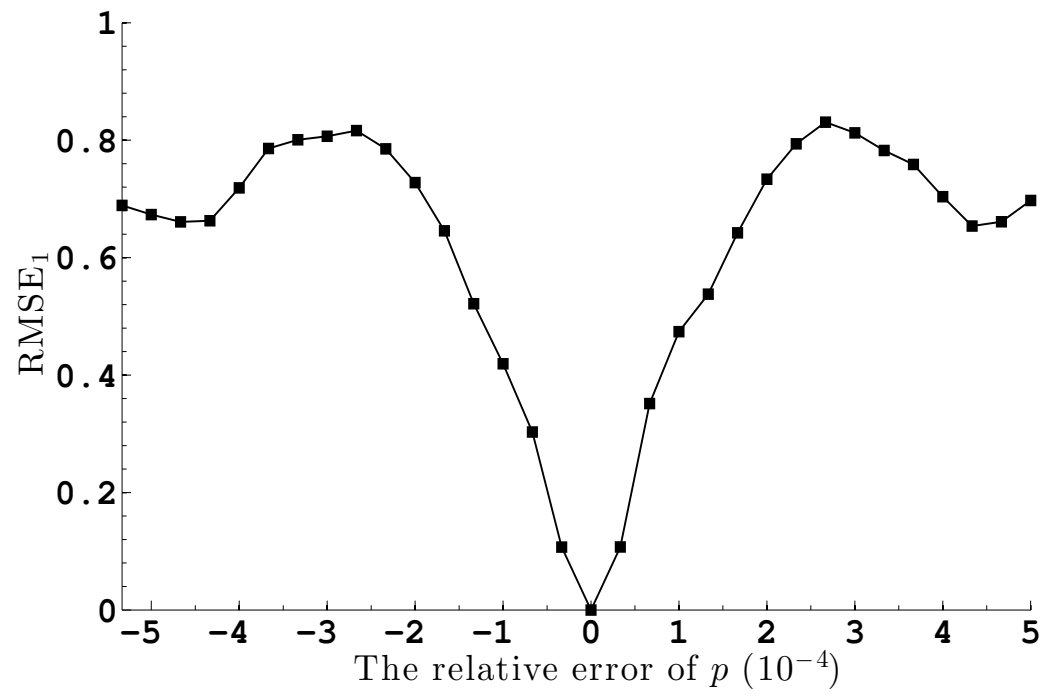

Figure 6. Variations of the $\mathrm{RMSE}_{1}$ versus the relative error of $p$ for the decryption system.

We have tested the performance of the proposed encryption-decryption system when the encrypted image is corrupted by noise or occlusion [39]. The decrypted images presented in Figs. 7(a) and 7(b), correspond to the images retrieved by the decryption system when the encrypted image of Fig. 5(b) is perturbed by additive and multiplicative Gaussian white noise with zero mean and variance of $\sigma^{2}=0.2$, respectively. The RMSEs between the original image (Fig. 5(a)) and the decrypted images (Figs. 7(a) and 7(b)) are 0.251 and 0.238 , respectively. If the encrypted image of Fig. 5(b) is occluded by $12.5 \%$ (Fig. $7(\mathrm{c})$ ) and $25 \%$ (Fig. $7(\mathrm{~d})$ ) of its area (the values of occluded pixels are replaced with the value of zero), we obtain the decrypted images depicted in Figs. 7(e) and $7(\mathrm{f})$, respectively. The RMSEs between the original image (Fig. 5(a)) and the decrypted images (Figs. $7(\mathrm{e})$ and $7(\mathrm{f})$ ) are 0.346 and 0.406 , respectively. Despite the 
loss quality that affects the decrypted images shown in Figs. 7(a), 7(b), 7(e), and 7(f), the presence of the original image (Fig. 5(a)) can be recognized in all of them. These examples show the robustness of the proposed encryption-decryption system to certain amount of degradation in the encrypted image by noise or occlusion.

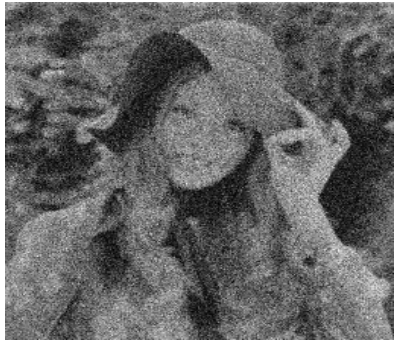

(a)

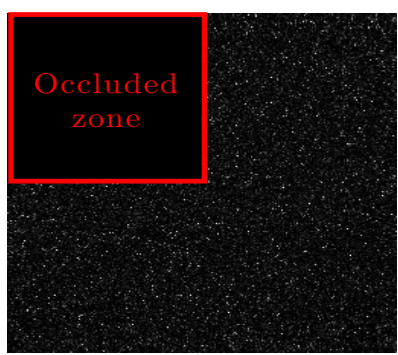

(d)

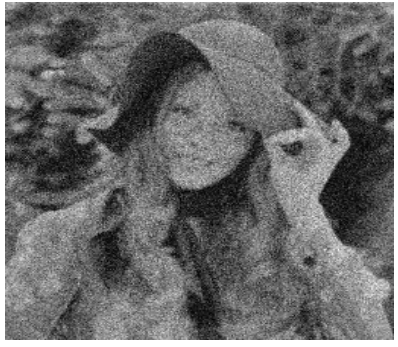

(b)

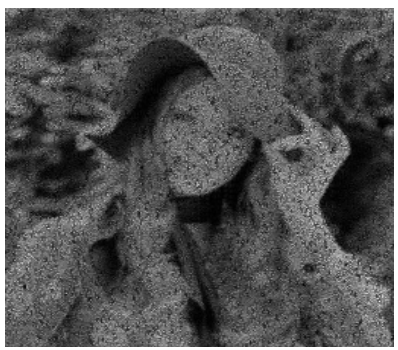

(e)

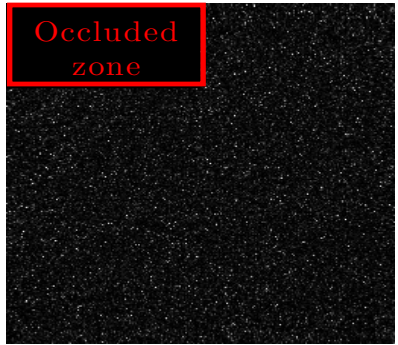

(c)

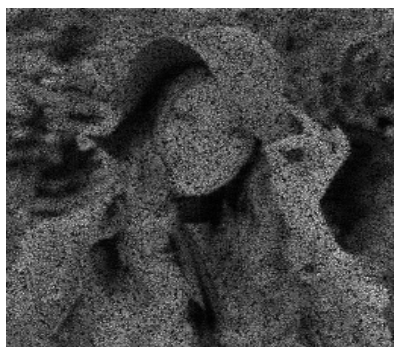

(f)

Figure 7. Decrypted images when the encrypted image of Fig. 5(b) is corrupted by a Gaussian white noise with zero mean and variance of $\sigma^{2}=0.2$ : (a) additive noise and (b) multiplicative noise. Occluded encrypted images from Fig. 5(b) with the following percentage occlusion of its area: (c) $12.5 \%$ and (d) $25 \%$. Decrypted images corresponding to the occluded encrypted images of: (e) Fig. 7(c) and (f) Fig. 7(d).

Finally, we propose some guidelines in order to increase the security of the JTCbased encryption system against the CPA [26], and KPA [27]. The nonlinear operation introduced in the JFPS already improves the security of the encryption system against the CPA, just as it was proved in $[14,16]$. To increase the security of the encryption system against KPA, we recommend to use different probability density functions (not only the uniform distribution) for the random code functions corresponding to the RPM $h(x)[14,16]$. A random complex mask (RCM) was utilized as key for the encryptiondecryption system presented in [16]. This RCM can be used to further improve the resistance of the JTC-based encryption in the FrFD against KPA [16].

3.2.1. Shift-invariance property of the RPM $h(x)$ in the decryption system If the RPM $h(x)$ is shifted to $x=-b$ with fractional order $\alpha$ in the initial step of the decryption system, the following result is obtained 


$$
\begin{aligned}
d_{\alpha}^{N_{3}}(u)= & e_{\alpha}^{N_{2}}(u) \mathscr{F}^{\alpha}\left\{\mathscr{T}_{-b ; \alpha}[h(x)]\right\}=e_{\alpha}^{N_{2}}(u) h_{\alpha}(u) \exp \{-i 2 \pi b u \csc \alpha\} \\
= & \frac{h_{\alpha}(u)}{\left|h_{\alpha}(u)\right|} \frac{h_{\alpha}(u)}{\left|h_{\alpha}(u)\right|} \exp \left\{i \pi u^{2} \cot \alpha\right\} \\
& \times g_{\alpha}^{*}(u) \exp \left\{-i \pi u^{2} \cot \alpha\right\} \exp \{i 2 \pi(-2 a-b) u \csc \alpha\} \\
& +\frac{h_{\alpha}(u) h_{\alpha}^{*}(u)}{\left|h_{\alpha}(u)\right|^{2}} g_{\alpha}(u) \exp \{i 2 \pi(2 a-b) u \csc \alpha\}
\end{aligned}
$$

The FrFT at fractional order $-\alpha$ of Eq. (15) is

$$
\begin{aligned}
d^{N_{3}}(x) & =\mathscr{F}^{-\alpha}\left\{d_{\alpha}^{N_{3}}(u)\right\} \\
& =\mathscr{T}_{-2 a-b ; \alpha}\left[\left\{h_{1}(x) *_{\alpha} h_{1}(x)\right\} \circledast_{\alpha} g(x)\right]+\mathscr{T}_{2 a-b ; \alpha}[g(x)] .
\end{aligned}
$$

When the absolute value is applied to the second term of Eq. (16), the decrypted image can be recovered at coordinate $x=2 a-b$

$$
\left|\mathscr{T}_{2 a-b ; \alpha}[g(x)]\right|=\left|\mathscr{T}_{2 a-b ; \alpha}[f(x) r(x)]\right|=f(x-2 a+b) .
$$

The Eq. (17) demonstrates that the encryption-decryption proposed in this paper preserves the shift-invariance property of the RPM $h(x)$ for decryption and the retrieval of the original image; this shift-invariance of the encryption-decryption system is a consequence of the fractional traslation invariance of the fractional convolution and fractional correlation operators (see Appendix C) [34].

3.2.2. Description of the optical setup Figure 8 shows the schematic representation of the optical setup for the proposed encryption system in the approach II. The encrypted image given by Eq. (11) can be optically implemented using a two-step JTC [35, 40, 41] in the FrFD. In the first step, the functions $\mathscr{T}_{-a ; \alpha}[h(x)]$ and $\mathscr{T}_{a ; \alpha}[g(x)]$ are sequentially displayed on the input plane of the setup. The intensity function $\left|h_{\alpha}(u)\right|^{2}$ is captured in the output plane of the encryption system when the function $\mathscr{T}_{-a ; \alpha}[h(x)]$ is placed at the input plane of the JTC and the optical FrFT is performed. Thereupon, the other intensity function $\left|g_{\alpha}(u)\right|^{2}$ is captured when the function $\mathscr{T}_{a ; \alpha}[g(x)]$ is placed at the input plane. Finally, the $\operatorname{JFPS}_{\alpha}(u)$ represented by Eq. (3) is captured in the second step [42]; in this step, the functions $\mathscr{T}_{-a ; \alpha}[h(x)]$ and $\mathscr{T}_{a ; \alpha}[g(x)]$ are simultaneously placed at the input plane of the JTC. Afterwards, the terms $\left|g_{\alpha}(u)\right|^{2}$ and $\left|h_{\alpha}(u)\right|^{2}$ are digitally subtracted from the JFPS, and then, this result is divided by $\left|h_{\alpha}(u)\right|^{2}$, and thus, the encrypted image of Eq. (11) is computed. This encrypted image is the only information to be transmitted; therefore, this system does not increase the amount of data to transmit prior the decryption system [14, 16, 17]. The optical FrFT can be performed by means of the optoelectronic setup developed in [43]. The fractional order $\alpha$ of the FrFT is defined by the distances $d_{1}, d_{2}$ and the focal length of the lens [28,36].

The pure linear phase terms contained in $\mathscr{T}_{a ; \alpha}[g(x)]$ and $\mathscr{T}_{-a ; \alpha}[h(x)]$ that are symmetrically introduced in the input plane of the JTC, see Eq. (2), can be implemented using an optical biprism or a phase-only spatial light modulator (SLM). The RPMs 
$r(x)$ and $h(x)$ can be implemented using a simple diffuser glass [12, 14]. In fact, these pure linear phase terms and the RPMs $r(x)$ and $h(x)$ can be displayed all together by means of a phase-only SLM. Following the sampling theorem for fractional bandlimited signals $[34,44]$, the sampling intervals in the spatial and fractional frequency domains of the encryption system have to be selected according to the fractional order $\alpha$, so that aliasing in the decrypted image can be avoided. This criterion has an effect when considering the pixelated devices involved in the setup: the CCD array sensor of the camera, the SLM display of the input plane of the FrFD-JTC (encryption system), and the SLM displays of the input plane and the FrFD plane in the processor for decryption.

The decryption system for the approach II is presented in the part II of Fig. 1, placing the functions: $\mathscr{T}_{-a ; \alpha}[h(x)]$ at the input plane of the JTC and $e_{\alpha}^{N_{2}}(u)$ in the FrFD.

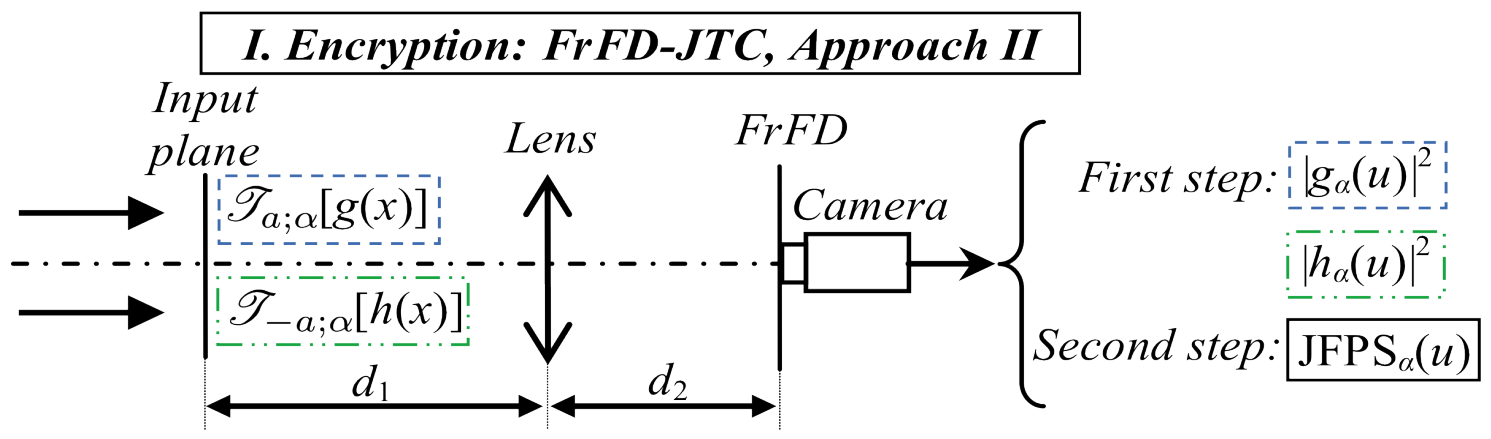

Figure 8. Schematic representation of the proposed optical encryption setup in approach II.

\section{Conclusion}

We have proposed a generalization of the encryption system based on DRPE and a JTC by using the following fractional Fourier operators: the FrFT, fractional traslation, fractional convolution and fractional correlation. An additional key given by the fractional order of the FrFT was introduced with respect to the JTC-based encryption system in the Fourier transform domain, this new key improves the security of the encryption system. The modification of the JFPS by means of a nonzero-order JTC in the FrFD and the introduction of a nonlinear operation on this modified JFPS has allowed for the retrieval of the original image with a higher image quality. The two approaches presented to improve the quality of the decrypted image do not increase the amount of data to be sent prior to the decryption stage. The encryption-decryption system proposed in this work preserves the shift-invariance property of the RPM $h(x)$ for the decryption system and the retrieval of the original image, which has been a big problem in the common definitions of fractional correlation and fractional convolution. Finally, the proposed encryption and decryption systems are suitable for optoelectronic 
implementation; a two-step JTC in the FrFD can be used for the encryption system and two successive optical fractional Fourier transforms for the decryption system.

\section{Acknowledgments}

This research has been partly funded by the Spanish Ministerio de Ciencia e Innovación and Fondos FEDER (Project DPI2009-08879). The first author also wishes to thank the Departamento Administrativo de Ciencia, Tecnología e Innovación from Colombia, COLCIENCIAS, for a doctoral scholarship.

\section{Appendix A. The fractional Fourier transform operator}

The fractional Fourier transform (FrFT) of order $\alpha$, is a linear integral operator that maps a given function $f(x)$ onto function $f_{\alpha}(u)$, by [28]

$$
f_{\alpha}(u)=\mathscr{F}^{\alpha}\{f(x)\}=\int_{-\infty}^{+\infty} f(x) K_{\alpha}(u, x) \mathrm{d} x,
$$

with

$$
\begin{aligned}
& K_{\alpha}(u, x)=C_{\alpha} \exp \left\{-i \pi\left[\left(u^{2}+x^{2}\right) \cot \alpha-2 u x \csc \alpha\right]\right\}, \\
& C_{\alpha}=\frac{\exp \left\{i\left(\frac{\pi}{4} \operatorname{sgn}(\alpha)-\frac{\alpha}{2}\right)\right\}}{\sqrt{|\sin \alpha|}},-\pi<\alpha \leq \pi, \quad \alpha=\frac{p \pi}{2}, \quad-2<p \leq 2,
\end{aligned}
$$

where $K_{\alpha}$ is the fractional Fourier kernel and sgn is the sign function. For $\alpha=0(p=0)$, it corresponds to the identity transform. For $\alpha=\pi / 2(p=1)$, it reduces to the direct standard Fourier transform. For $\alpha=\pi(p=2)$, the reverse transform is obtained. For $\alpha=-\pi / 2(p=-1)$, it corresponds to the inverse standard Fourier transform. The inverse FrFT corresponds to the FrFT at fractional order $-\alpha$. The FrFT operator is additive with respect to the fractional order, $\mathscr{F}^{\alpha} \mathscr{F}^{\beta}=\mathscr{F}^{\alpha+\beta}$.

The optical FrFT can be implemented using the schematic representation presented in Fig. 8, where the fractional order $\alpha$ is defined in terms of the distances $d_{1}, d_{2}$ and the focal length $\left(f_{l}\right)$ of the lens $[28,36]$

$$
\alpha=\arccos \left(\frac{\sqrt{\left(f_{l}-d_{1}\right)\left(f_{l}-d_{2}\right)}}{f_{l}}\right) .
$$

\section{Appendix B. The fractional traslation operator}

We use the notion of the fractional traslation introduced in Ref. [34], which defines the fractional traslation operator of order fractional $\alpha$ and real value $\tau$ as

$$
\mathscr{T}_{\tau ; \alpha} f(x)=f(x-\tau) \exp \left\{i 2 \pi \tau\left(x-\frac{\tau}{2}\right) \cot \alpha\right\} .
$$

For a given $\alpha$, the fractional traslation operator $\mathscr{T}_{\tau ; \alpha}$ forms a commutative group. The composition law is $\mathscr{T}_{\tau_{1} ; \alpha} \mathscr{T}_{\tau_{2} ; \alpha}=\mathscr{T}_{\tau_{1}+\tau_{2} ; \alpha}$. When the fractional order $\alpha$ is equal 
to $\pi / 2$, the fractional traslation operator is reduced to the usual traslation operator $\mathscr{T}_{\tau ; \pi / 2} f(x)=\mathscr{T}_{\tau} f(x)=f(x-\tau)$. The FrFT at fractional order $\alpha$ of Eq. (B.1) is

$$
\mathscr{F}^{\alpha}\left\{\mathscr{T}_{\tau ; \alpha} f(x)\right\}=f_{\alpha}(u) \exp \{i 2 \pi \tau u \csc \alpha\} .
$$

\section{Appendix C. The fractional convolution and fractional correlation operators}

The definitions of the fractional convolution and fractional correlation operators that are used in the encryption-decryption systems of Sections 2 and 3 were proposed in Ref. [34].

The fractional convolution operator is defined by

$$
f(x) *_{\alpha} g(x)=\int_{-\infty}^{+\infty} f(z) g(x-z) \exp \{i 2 \pi z(x-z) \cot \alpha\} \mathrm{d} z .
$$

Using the FrFTs $\mathscr{F}^{\alpha}\{f(x)\}=f_{\alpha}(u)$ and $\mathscr{F}^{\alpha}\{g(x)\}=g_{\alpha}(u)$, the Eq. (C.1) can be expressed as

$$
f(x) *_{\alpha} g(x)=\mathscr{F}^{-\alpha}\left[f_{\alpha}(u) g_{\alpha}(u) \exp \left\{i \pi u^{2} \cot \alpha\right\}\right] .
$$

We have the following special cases $f(x) *_{\pi / 2} g(x)=f(x) * g(x)$, which is the usual convolution operation and $f(x) *_{0} g(x)=f(0) g(0) \delta(x)$, where $\delta(x)$ is the Dirac delta distribution. The fractional convolution of a function $f(x)$ and a shifted Dirac delta function $\delta(x-\tau)$ is

$$
f(x) *_{\alpha} \delta(x-\tau)=f(x-\tau) \exp \{i 2 \pi \tau(x-\tau) \cot \alpha\},
$$

therefore, the fractional traslation operator can be expressed in terms of a fractional convolution

$$
\mathscr{T}_{\tau ; \alpha} f(x)=\left[f(x) *_{\alpha} \delta(x-\tau)\right] \exp \left\{i \pi \tau^{2} \cot \alpha\right\} .
$$

The fractional convolution operator is fractional traslation invariant

$$
\mathscr{T}_{\tau ; \alpha}\left[f(x) *_{\alpha} g(x)\right]=\mathscr{T}_{\tau ; \alpha}[f(x)] *_{\alpha} g(x)=f(x) *_{\alpha} \mathscr{T}_{\tau ; \alpha}[g(x)],
$$

the previous equation is a straightforward generalization of the traslation invariance of the usual convolution operation.

The fractional correlation operator is given by

$$
f(x) \circledast_{\alpha} g(x)=\int_{-\infty}^{+\infty} f(z) g^{*}(z-x) \exp \{-i 2 \pi x(z-x) \cot \alpha\} \mathrm{d} z .
$$

Using the FrFTs $\mathscr{F}^{\alpha}\{f(x)\}=f_{\alpha}(u)$ and $\mathscr{F}^{\alpha}\{g(x)\}=g_{\alpha}(u)$, the integral form of fractional correlation can be expressed as

$$
f(x) \circledast_{\alpha} g(x)=\mathscr{F}^{-\alpha}\left[f_{\alpha}(u) g_{\alpha}^{*}(u) \exp \left\{-i \pi u^{2} \cot \alpha\right\}\right] .
$$


The special cases for the fractional correlation operator are $f(x) \circledast_{\pi / 2} g(x)=f(x) \circledast$ $g(x)$, which represents the usual correlation operation and $f(x) \circledast_{0} g(x)=f(0) g^{*}(0) \delta(x)$.

Finally, the fractional correlation operator is also fractional traslation invariant

$$
\mathscr{T}_{\tau ; \alpha}\left[f(x) \circledast_{\alpha} g(x)\right]=\mathscr{T}_{\tau ; \alpha}[f(x)] \circledast_{\alpha} g(x)=f(x) \circledast_{\alpha} \mathscr{T}_{-\tau ; \alpha}[g(x)] .
$$

The Eq. (C.8) is a generalization of the traslation invariance of the usual correlation operation.

\section{References}

[1] Pérez-Cabré E and Millán M S 2011 Optical data encryption Optical and Digital Image Processing: Fundamentals and Applications ed G Cristóbal, P Schelkens and H Thienpont (New York: Wiley-VCH)

[2] Réfrégier P and Javidi B 1995 Optical image encryption based on input plane and Fourier plane random encoding Opt. Lett. 20 767-769

[3] Matoba O and Javidi B 1999 Encrypted optical memory system using three-dimensional keys in the Fresnel domain Opt. Lett. 24 762-764

[4] Situ G and Zhang J 2004 Double random phase encoding in the Fresnel domain Opt. Lett. 29 $1584-1586$

[5] Unnikrishnan G, Joseph J and Singh K 2000 Optical encryption by double-random phase encoding in the fractional Fourier domain Opt. Lett. 25 887-889

[6] Liu S, Yu L and Zhu B 2001 Optical image encryption by cascaded fractional Fourier transforms with random phase filtering Opt. Comm. 187 57-63

[7] Nishchal N K, Joseph J and Singh K 2003 Fully phase encryption using fractional Fourier transform Opt. Eng. 42 1583-1588

[8] Nishchal N K, Joseph J and Singh K 2004 Fully phase-encrypted memory using cascaded extended fractional Fourier transform Opt. Lasers Eng. 42 141-151

[9] Singh N and Sinha A 2008 Optical image encryption using fractional Fourier transform and chaos Opt. Lasers Eng. 46 117-123

[10] Goodman J W 1996 Introduction to Fourier Optics 2nd edn (New York: McGraw-Hill)

[11] Nomura T and Javidi B 2000 Optical encryption using a joint transform correlator architecture Opt. Eng. 39 2031-2035

[12] Rueda E, Barrera J F, Henao R and Torroba R 2009 Optical encryption with a reference wave in a joint transform correlator architecture Opt. Comm. 282 3243-3249

[13] Barrera J F, Tebaldi M, Ríos C, Rueda E, Bolognini N and Torroba R 2012 Experimental multiplexing of encrypted movies using a JTC architecture Opt. Express 20 3388-3393

[14] Vilardy J M, Millán M S and Pérez-Cabré E 2013 Improved decryption quality and security of a joint transform correlator-based encryption system J. Opt. 15025401

[15] Nomura T, Mikan S, Morimoto Y and Javidi B 2003 Secure Optical Data Storage with Random Phase Key Codes by use of a Configuration of a Joint Transform Correlator Appl. Opt. 42 $1508-1514$

[16] Vilardy J M, Millán M S and Pérez-Cabré E 2014 Nonlinear optical security system based on a joint transform correlator in the Fresnel domain Appl. Opt. 53 1674-1682

[17] Vilardy J M, Millán M S and Pérez-Cabré E 2013 Joint transform correlator-based encryption system using the Fresnel transform and nonlinear filtering Proc. of SPIE $\mathbf{8 7 8 5} 87853 \mathrm{~J}$

[18] Lu D and Jin W 2011 Color image encryption based on joint fractional Fourier transform correlator Opt. Eng. 50068201

[19] Rajput S K and Nishchal N K 2012 Image encryption and authentication verification using fractional nonconventional joint transform correlator Opt. Lasers Eng. 50 1474-1483 
[20] Wang Q, Guo Q, Lei L and Zhou J 2013 Optical image encryption based on joint fractional transform correlator architecture and digital holography Opt. Eng. 52048201

[21] Wang Q, Guo Q, Lei L and Zhou J 2014 Iterative partial phase encoding based on joint fractional Fourier transform correlator adopting phase-shifting digital holography Opt. Comm. 313 1-8

[22] Lu D and Jin W 2011 Fully phase color image encryption based on joint fractional Fourier transform correlator and phase retrieval algorithm Chinese Opt. Lett. 9021002

[23] Carnicer A, Montes-Usategui M, Arcos S and Juvells I 2005 Vulnerability to chosen-cyphertext attacks of optical encryption schemes based on double random phase keys Opt. Lett. $301644-$ 1646

[24] Frauel Y, Castro A, Naughton T J and Javidi B 2007 Resistance of the double random phase encryption against various attacks," Opt. Express 15 10253-10265

[25] Peng X, Zhang P, Wei H and Yu B 2006 Known-plaintext attack on optical encryption based on double random phase keys Opt. Lett. 31 1044-1046

[26] Barrera J F, Vargas C, Tebaldi M and Torroba R 2010 Chosen-plaintext attack on a joint transform correlator encrypting system Opt. Commun. 283 3917-3921

[27] Barrera J F, Vargas C, Tebaldi M, Torroba R and Bolognini N 2010 Known-plaintext attack on a joint transform correlator encrypting system Opt. Lett. 35 3553-3555

[28] Ozaktas H M, Zalevsky Z and Kutay M A 2001 The Fractional Fourier Transform with Applications in Optics and Signal Processing (New York: Wiley-VCH)

[29] Chen W, Javidi B and Chen X 2014 Advances in optical security systems Adv. Opt. Photon. 6 $120-155$

[30] Chen W, Chen X, Stern A and Javidi B 2013 Phase-modulated optical system with sparse representation for information encoding and authentication IEEE Photonics Journal 56900113

[31] Pérez-Cabré E, Cho M and Javidi B 2011 Information authentication using photon-counting double-random-phase encrypted images Opt. Lett. 36 22-24

[32] Pérez-Cabré E, Abril H C, Millán M S and Javidi B 2012 Photon-counting double-random-phase encoding for secure image verification and retrieval J. Opt. 14094001

[33] Markman A and Javidi B 2014 Full-phase photon-counting double-random-phase encryption $J$. Opt. Soc. Am. A 31 394-403

[34] Torres R, Pellat-Finet P and Torres Y 2010 Fractional convolution, fractional correlation and their translation invariance properties Signal Processing 90 1976-1984

[35] Li C-T, Yin S and Yu F T S 1998 Nonzero-order joint transform correlator Opt. Eng. 37 58-65

[36] Pellat-Finet P 1994 Fresnel diffraction and the fractional-order Fourier transform, Opt. Lett. 19, $1388-1390$

[37] Gonzalez R C, Woods R E and Eddins S L 2009 Digital Image Processing Using Matlab 2nd edn (USA: Gatesmark Publishing)

[38] Millán M S 2012 Advanced optical correlation and digital methods for pattern matching-50th anniversary of Vander Lugt matched filter J. Opt. 14103001

[39] Goudail F, Bollaro F, Javidi B and Réfrégier P 1998 Influence of a perturbation in a double phase-encoding system J. Opt. Soc. Am. A 15 2629-2638

[40] Pérez E, Chałasińska-Macukow K, Styczyński K, Kotyński R and Millán M S 1997 Dual nonlinear correlator based on computer controlled joint transform processor: Digital analysis and optical results J. Mod. Opt. 44 1535-1552

[41] Pérez E, Millán M S and Chałasińska-Macukow K 2002 Optical pattern recognition with adjustable sensitivity to shape and texture Opt. Commun. 202 239-255

[42] Tebaldi M, Horrillo S, Pérez-Cabré E, Millán M S, Amaya D, Torroba R and Bolognini N 2011 Experimental color encryption in a joint transform correlator architecture J. Physics: Conf. Ser. 274012054

[43] Rodrigo J A, Alieva T and Calvo M L 2009 Programmable two-dimensional optical fractional Fourier processor Opt. Express 17 4976-4983

[44] Torres R, Pellat-Finet P and Torres Y 2006 Sampling theorem for fractional bandlimited signals: 
a self-contained proof. Application to digital holography IEEE Sig. Process. Lett. 13 676-679 